\title{
MEAN SUMMABILITY METHODS FOR LAGUERRE SERIES
}

\author{
KRZYSZTOF STEMPAK
}

ABSTRACT. We apply a construction of generalized convolution in

$$
L^{1}\left(\mathbb{R}_{+} \times \mathbb{R}, x^{2 \alpha-1} d x d t\right), \quad \alpha \geq 1,
$$

cf. [8], to investigate the mean convergence of expansions in Laguerre series. Following ideas of $[4,5]$ we construct a functional calculus for the operator

$$
L=-\left(\frac{\partial^{2}}{\partial x^{2}}+\frac{2 \alpha-1}{x} \frac{\partial}{\partial x}+x^{2} \frac{\partial^{2}}{\partial t^{2}}\right), \quad x>0, t \in \mathbb{R}, \alpha \geq 1 .
$$

Then, arguing as in [3], we prove results concerning the mean convergence of some summability methods for Laguerre series. In particular, the classical AbelPoisson and Bochner-Riesz summability methods are included.

\section{INTRODUCTION}

The problem of mean convergence of Laguerre series has been investigated by a number of authors. The most celebrated result in this direction, proved by Askey and Wainger in 1965 [1] claims the following:

Let $a \geq 0,1 \leq p \leq \infty$ and for any function $f \in L^{p}\left(\mathbb{R}_{+}, d x\right)$ define

$$
c_{n}=c_{n}(f)=\int_{0}^{\infty} f(x) \mathscr{L}_{n}^{a}(x) d x .
$$

Then

$$
\lim _{k \rightarrow \infty}\left\|f-\sum_{n=0}^{k} c_{n} \mathscr{L}_{n}^{a}\right\|_{L^{p}(d x)}=0,
$$

for every $f \in L^{p}\left(\mathbb{R}_{+}, d x\right)$ if and only if $4 / 3<p<4$. Here $\mathscr{L}_{n}^{a}, n=$ $0,1,2, \ldots$, denotes the $n$th Laguerre function

$$
\mathscr{L}_{n}^{a}(x)=(n ! / \Gamma(n+a+1))^{1 / 2} e^{-x / 2} x^{a / 2} L_{n}^{a}(x),
$$

$L_{n}^{a}(x), n=0,1,2, \ldots$, being the Laguerre polynomials of order $a>-1$ defined by

$$
\sum_{n=0}^{\infty} t^{n} L_{n}^{a}(x)=(1-t)^{-a-1} \exp (x t /(t-1)) .
$$

Received by the editors December 30, 1987 and, in revised form, December 22, 1988.

1980 Mathematics Subject Classification (1985 Revision). Primary 43A55, 42C15.

Key words and phrases. Laguerre expansions, generalized convolution, mean convergence. 
In 1969 Muckenhoupt [7] established the following result concerning the Abel-Poisson summability method for Laguerre expansions:

Let $a>-1$ and for any function $f \in L^{p}\left(\mathbb{R}_{+}, x^{a} e^{-x} d x\right)$ define

$$
d_{n}=d_{n}(f)=\frac{n !}{\Gamma(n+\alpha+1)} \int_{0}^{\infty} f(x) L_{n}^{a}(x) x^{a} e^{-x} d x .
$$

Then, for every $f \in L^{p}\left(\mathbb{R}_{+}, x^{a} e^{-x} d x\right), 1 \leq p \leq \infty$,

$$
\lim _{r \rightarrow 1^{-}}\left\|f-\sum_{n=0}^{\infty} r^{n} d_{n} L^{a}\right\|_{L^{p}\left(x^{a} e^{-x} d x\right)}=0 .
$$

Recently, Dlugosz [3] investigated mean as well as almost everywhere convergence of some summability methods for Laguerre expansions. Under some mild condition on a summability kernel $K, K: \mathbb{R}_{+} \rightarrow \mathbb{C}$, she proved that for every $f \in L^{p}\left(\mathbb{R}_{+}, d x\right), 1 \leq p<\infty$,

$$
\lim _{t \rightarrow 0}\left\|f-\sum_{n=0}^{\infty} K(t n) c_{n} \mathscr{L}_{n}^{a}\right\|_{L^{p}(d x)}=0,
$$

$a \in \mathbb{N}$ being an arbitrary nonnegative integer.

This restriction on $a$ is motivated by the approach used in [3]. Roughly, the method is based on the fact that the Laguerre functions of an integral order $a=$ $0,1,2, \ldots$, appear (nearly) as eigenfunctions of the sublaplacian, an operator acting on the $2 a+3$ dimensional Heisenberg group.

The present paper follows ideas of an earlier version of [3] and, in some sense, fills out the gap caused by the restriction $a \in \mathbb{N}$. More precisely, we prove that for any $a \geq 0, f \in L^{p}\left(\mathbb{R}_{+}, x^{a} d x\right), 1 \leq p<\infty$,

$$
\lim _{t \rightarrow 0} \| f-\sum_{n=0}^{\infty} K(\text { tn }) b_{n} l_{n}^{a} \|_{L^{p}\left(x^{a} d x\right)}=0,
$$

where

$$
l_{n}^{a}(x)=(n ! / \Gamma(n+a+1))^{1 / 2} e^{-x / 2} L_{n}^{a}(x)
$$

and $b_{n}, n=0,1,2, \ldots$, are given by

$$
b_{n}=b_{n}(f)=\int_{0}^{\infty} f(x) l_{n}^{a}(x) x^{a} d x,
$$

and, for instance, the summability kernel $K, K: \mathbb{R}_{+} \rightarrow \mathbb{C}$, may be taken to be $K(\lambda)=\exp (-\lambda)$ or $K(\lambda)=(1-\lambda)_{+}^{N}, N>a+5$.

Our technique is based on [8] where the author defined a product in $L^{1}\left(X, x^{2(x-1} d x d t\right), X=\mathbb{R}_{+} \times \mathbb{R}, \alpha \geq 1$, called the generalized convolution, turning this space into a commutative Banach algebra. We mention that the underlying differential operator is

$$
L=-\left(\frac{\partial^{2}}{\partial x^{2}}+\frac{2 \alpha-1}{x} \frac{\partial}{\partial x}+x^{2} \frac{\partial^{2}}{\partial t^{2}}\right)
$$


In case $\alpha=m, m=1,2,3, \ldots$, the product in $L^{1}\left(X, x^{2 \alpha-1} d x d t\right)$ comes from an algebra (under the ordinary convolution) of so-called radial integrable functions on the $2 m+1$ dimensional Heisenberg group and $L$ is then the radial part of sublaplacian there.

The Gelfand transform in $L^{1}\left(X, x^{2 \alpha-1} d x d t\right)$ is described by means of eigenfunctions of $L$ and, as it appears, the functions $l_{n}^{\alpha-1}, n=0,1,2,3, \ldots$, are involved here. This gave rise, basically, to an attempt to follow [3]. We should also emphasize that ideas of Hulanicki [4] and Hulanicki and Jenkins [5] play here, as well as in [3], the crucial role. In particular, we follow [4] rather closely to get Lemma 2.5 and then [5] to construct a functional calculus described in Theorem 4.3 (cf. [5, Theorem 1.12]). Although there is no underlying group structure (for arbitrary $\alpha \geq 1$ ), the generalized convolution is a sufficient tool that allows us to substitute for it.

At the end we state a ew remarks concerning the presentation. Since the case $\alpha=1$ should be treated separately (this is since the definitions (1.1) and (1.2) for $\alpha=1$ are slightly different than those for $\alpha>1$, cf. [8]), we decided, for simplicity, to consider the case $\alpha>1$ only. Nevertheless, all results, especially those of $\S 5$, are still valid when $\alpha=1$.

We mention also that $C$ will be used to denote a constant which may vary from line to line.

Finally, the author would like to thank Jacek Cygan for a helpful remark concerning the proof of Lemma 3.1.

\section{Preliminaries}

We recall a construction of a commutative Banach algebra originated in [8]. Let $\alpha>1$ be a fixed real number. We equip the space $X=\mathbb{R}_{+} \times \mathbb{R}$ with the measure $d \mu(x, t)=x^{2 \alpha-1} d x d t$ and by $L^{p}(\mu)$ we denote the corresponding Lebesgue spaces endowed with the norms

$$
\|f\|_{p}=\left(\int_{X}|f|^{p} d \mu\right)^{1 / p}
$$

We will denote elements of $X$ using the Greek letters $\xi$ and $\eta$. For $\xi=(x, t)$, $\eta=(y, u)$, and $\theta, \varphi \in \mathbb{R}$, we define the product $(\xi, \eta)_{\theta, \varphi}$ by

$$
(\xi, \eta)_{\theta, \varphi}=\left(\left(x^{2}+y^{2}-2 x y \cos \theta\right)^{1 / 2}, t-u+x y \cos \varphi \sin \theta\right) .
$$

Next, we define the probability measure $\nu$ on $[0, \pi] \times[0, \pi]$ by

$$
d \nu(\theta, \varphi)=\frac{\alpha-1}{\pi}(\sin \varphi)^{2 x-3}(\sin \theta)^{2 x-2} d \theta d \varphi .
$$

Finally, the generalized translation operators $T^{\eta}, \eta \in X$, and the convolution $f * g, f, g \in L^{1}(\mu)$, are given by

$$
T^{\eta} f(\xi)=\int_{0}^{\pi} \int_{0}^{\pi} f\left((\xi, \eta)_{\theta, \varphi}\right) d \nu(\theta, \varphi), \quad f \in L^{p}(X),
$$


and

$$
f * g(\xi)=\int_{X} T^{\eta} f(\xi) g(\eta) d \mu(\eta) .
$$

In [8] the author proved that $L^{1}(\mu)$ equipped with the convolution (1.4) is a commutative Banach algebra. Furthermore, $L^{1}(\mu)$ admits a natural *structure given by $f^{*}(\xi)=\overline{f(\bar{\xi})}$, where $\bar{\xi}=(x,-t)$ for $\xi=(x, t)$. Moreover, the operators $T^{\eta}, \eta \in X$, are submarkovian contractions on every $L^{p}(\mu)$, $\|f * g\|_{p} \leq\|f\|_{1}\|g\|_{p}, \quad 1 \leq p \leq \infty$, and $\left(T^{\eta}\right)^{*}=T^{\bar{\eta}}$ on $L^{2}(\mu)$. It has been also remarked that an underlying operator is the following second-order differential operator

$$
L=-\left(\frac{\partial^{2}}{\partial x^{2}}+\frac{2 \alpha-1}{x} \frac{\partial}{\partial x}+x^{2} \frac{\partial^{2}}{\partial t^{2}}\right),
$$

which is positive, symmetric in $L^{2}(\mu)$, and homogeneous of degree 2 if $X$ is endowed with the family of dilations $\left(\delta_{r}\right)_{r>0}, \delta_{r}(x, t)=\left(r x, r^{2} t\right)$.

There are two series of eigenfunctions for $L$. Let $L_{n}^{a}(x), n=0,1,2, \ldots$, be the Laguerre polynomials of order $a>-1$ defined in terms of the generating function

$$
\sum_{n=0}^{\infty} t^{n} L_{n}^{a}(x)=(1-t)^{-a-1} \exp (x t /(t-1)) .
$$

For $\lambda \in \mathbb{R}, \lambda \neq 0$, and $n=0,1,2, \ldots$, we set

$$
\varphi_{\lambda, n}(x, t)=n ! \Gamma(\alpha) \Gamma(n+\alpha)^{-1} e^{i \lambda t} e^{-|\lambda| x^{2} / 2} L_{n}^{\alpha-1}\left(|\lambda| x^{2}\right) .
$$

Then, [8], $\left|\varphi_{\lambda, n}(x, t)\right|<1$ and

$$
L \varphi_{\lambda, n}=4|\lambda|(\alpha / 2+n) \varphi_{\lambda, n} .
$$

On the other hand, the functions

$$
\psi_{\tau}(x, t)=2^{\alpha-1} \Gamma(\alpha) J_{\alpha-1}(x \tau) /(x \tau)^{\alpha-1}, \quad \tau \geq 0,
$$

form the second series of eigenfunctions for $L$, namely $L \psi_{\tau}=\tau^{2} \psi_{\tau}$. Here $J_{a}$ denotes the Bessel function of order $a$. The eigenfunctions are kernels in a transform defined on $L^{1}(\mu)$. Let $f \in L^{1}(\mu)$. We define its transform $\hat{f}$ as follows: for any $\lambda \neq 0$ and $n=0,1,2, \ldots$,

$$
\hat{f}(\lambda, n)=\int_{X} f \varphi_{\lambda, n} d \mu
$$

and, for any $\tau \geq 0$,

$$
\hat{f}(\tau)=\int_{X} f \psi_{\tau} d \mu
$$

It has been proved in [8], that for any $f, g \in L^{1}(\mu)$

$$
(f * g)^{\widehat{ }}=\hat{f} \hat{g},
$$


and any multiplicative functional of the commutative Banach algebra $L^{1}(\mu)$ is of the form (1.8) or (1.9).

By using (1.7) and the symmetry of $L$ one can observe that

$$
(L f)^{\wedge}(\lambda, n)=4|\lambda|(\alpha / 2+n) \hat{f},
$$

for any function $f \in L^{1}(\mu)$, with $L f \in L^{1}(\mu)$. Next, by (1.10) and (1.11) (or directly from the definitions) we have

$$
L(f * g)=L f * g=f * L g,
$$

for any suitable pair of functions $f$ and $g$.

There is a heat kernel naturally associated to the operator $L$ (cf. [8]). More precisely, there exists a unique $C^{\infty}$-function $p(\xi, u)=p_{u}(\xi)$ on $X \times(0, \infty)$ with the following properties:

(i) $\left(\partial_{u}+L\right) p=0$ on $X \times(0, \infty)$;

(ii) $p(\xi, u) \geq 0, p_{u}(x,-t)=p_{u}(x, t), \int_{X} p_{u} d u=1$;

(iii) $p_{u_{1}} * p_{u_{2}}=p_{u_{1}+u_{2}}, u_{1}, u_{2}>0$;

(iv) $r^{-(2 \alpha+2)} p_{u}\left(\delta_{1 / r}(\xi)\right)=p_{r^{2} u}(\xi)$.

Furthermore, [8],

$$
\hat{p}_{u}(\lambda, n)=\exp (-4|\lambda|(\alpha / 2+n) u),
$$

and, for any $f \in L^{p}(\mu), 1 \leq p \leq \infty, F(u, \xi)=p_{u} * f(\xi)$ satisfies the equation

$$
\left(\partial_{u}+L\right) F=0 \text {. }
$$

Remark, that using the notation

$$
\rho_{r} f(\xi)=r^{-(2 \alpha+2)} f\left(\delta_{1 / r}(\xi)\right),
$$

the homogeneity property (iv) of the kernel $\left(p_{u}\right)_{u>0}$ may be written as

$$
\rho_{r^{1 / 2}} p_{1}=p_{r}, \quad r>0 .
$$

Observe also, that $\left(\rho_{r}\right)_{r>0}$ forms a one-parameter family of isometric isomorphisms of $L^{1}(\mu)$ and for any $k \in L^{1}(\mu)$ with $\int_{X} k(x) d \mu=1,\left(\rho_{r} k\right)_{r>0}$ is an approximate identity in $L^{p}(\mu), 1 \leq p<\infty$; that is, $\rho_{r} k * g \rightarrow g$ in $L^{p}(\mu)$ as $r \rightarrow 0$.

We endow the space $X$ with the homogeneous of degree one norm (with respect to $\left.\left(\delta_{r}\right)_{r>0}\right)$

$$
|\xi|=|(x, t)|=\left(x^{4}+4 t^{2}\right)^{1 / 4} .
$$

It is not difficult to verify that there is a measure $\sigma$ on the "unit sphere" $\{\xi \in$ $X:|\xi|=1\}$ such that for any $f \in L^{1}(\mu)$ the following polar decomposition formula holds

$$
\int_{X} f(\xi) d \mu(\xi)=\int_{0}^{\infty} \int_{\left|\xi^{\prime}\right|=1} f\left(\delta_{r} \xi^{\prime}\right) d \sigma\left(\xi^{\prime}\right) r^{2 \alpha+1} d r .
$$


Let $\mathscr{D}(X)$ denote the family of all restrictions on $X$ of compactly supported $C^{\infty}$-functions on $\mathbb{R}^{2}$, which are even with respect to the first variable. It might be easily seen that $\varphi * \psi \in \mathscr{D}(X)$ for any $\varphi, \psi \in \mathscr{D}(X)$ and, moreover, $f * \psi$ is a $C^{\infty}$-function for any $f \in L^{p}(X), 1 \leq p \leq \infty$, and $\psi \in \mathscr{D}(X)$. Further, we can construct a distribution theory on $X$, with underlying (commuting) operators $L$ and $\partial / \partial t$ and $\mathscr{D}(X)$ being test functions. Namely, we define the topology in $\mathscr{D}(X)$ by saying that $\psi_{j} \rightarrow \psi, \psi_{j}, \psi \in \mathscr{D}(X)$, provided that supp $\psi_{j} \subseteq K, K$ being a compact set in $\mathbb{R}^{2}$ and

$$
\sup _{(x, t) \in K}\left|P(L, \partial / \partial t) \psi_{j}(x, t)-\psi(x, t)\right| \rightarrow 0
$$

as $j \rightarrow \infty$, for any polynomial $P$ of two variables. By $\mathscr{D}^{\prime}(X)$ we then denote the set of all continuous linear functionals on $\mathscr{D}(X)$. Then $\langle F, \psi\rangle$ means the value of $F \in \mathscr{D}^{\prime}(X)$ on $\psi \in \mathscr{Z}(X)$. Next, for $F \in \mathscr{D}^{\prime}(X)$ we define $L F$ and $\partial F / \partial t$ by $\langle L F, \psi\rangle=\langle F, L \psi\rangle$ and $\langle\partial F / \partial t, \psi\rangle=-\langle F, \partial \psi / \partial t\rangle$. We also define the convolution $F * \psi, F \in \mathscr{D}^{\prime}(X), \psi \in \mathscr{D}(X)$, by $\langle F * \psi, \varphi\rangle=$ $\langle F, \psi * \varphi\rangle, \varphi \in \mathscr{D}(X)$. It might be observed that a long list of properties of the classic distributions in $\mathbb{R}^{n}$ remains valid also in our context. In particular, the formula (1.12) still holds if $f$ is replaced by any $F \in \mathscr{D}^{\prime}(X)$ and $g$ by any $\psi \in \mathscr{L}(X)$.

\section{WeIGHTED CONVOLUTION ALGEBRAS}

For the sake of convenience we denote $\tau(\xi)=|\xi|, \xi \in X$. Then we start with the following lemmas.

Lemma 2.1. For every $\xi, \eta \in X, \varphi, \theta \in \mathbb{R}$,

$$
\tau(\xi)-\tau(\eta) \leq \tau\left((\xi, \eta)_{\theta, \varphi}\right) \leq \tau(\xi)+\tau(\eta) .
$$

Proof. Consider the five-dimensional Heisenberg group $\mathbb{H}_{2}=\mathbb{R}^{2} \times \mathbb{R}^{2} \times \mathbb{R}$ with multiplication law

$$
\left(\bar{x}_{1}, \bar{y}_{1}, t_{1}\right)\left(\bar{x}_{2}, \bar{y}_{2}, t_{2}\right)=\left(\bar{x}_{1}+\bar{x}_{2}, \bar{y}_{1}+\bar{y}_{2}, t_{1}+t_{2}+\bar{x}_{1} \bar{y}_{2}-\bar{x}_{2} \bar{y}_{1}\right),
$$

$\bar{x}_{i}, \bar{y}_{i} \in \mathbb{R}^{2}$ and $\bar{x} \bar{y}=x_{1} y_{1}+x_{2} y_{2}$ being the Euclidean product in $\mathbb{R}^{2}$. Then $(\bar{x}, \bar{y}, t)^{-1}=(-\bar{x},-\bar{y},-t)$ and the norm

$$
|(\bar{x}, \bar{y}, t)|_{\mathrm{H}}=\left(\left(\|\bar{x}\|^{2}+\|\bar{y}\|^{2}\right)^{2}+4 t^{2}\right)^{1 / 4}
$$

$\left(\|\bar{x}\|=\left(x_{1}^{2}+x_{2}^{2}\right)^{1 / 2}, \bar{x}=\left(x_{1}, x_{2}\right)\right.$, denotes the Euclidean norm in $\left.\mathbb{R}^{2}\right)$ satisfies the triangle inequality (cf. [2])

$$
\left|\gamma_{1} \gamma_{2}\right|_{\mathrm{H}} \leq\left|\gamma_{1}\right|_{\mathrm{H}}+\left|\gamma_{2}\right|_{\mathrm{H}}, \quad \gamma_{i} \in \mathbb{H}_{2} .
$$

Now, for $\xi=(x, t), \eta=(y, u)$ consider $\gamma_{1}, \gamma_{2} \in \mathbb{H}_{2}$ given by $\gamma_{1}=(x, 0,0$, $0, t)$ and $\gamma_{2}=(y \cos \theta, 0, y \cos \varphi \sin \theta, y \sin \varphi \sin \theta, u)$. Straightforward calculation yields $\left|\gamma_{1}\right|_{\mathrm{H}}=|\xi|,\left|\gamma_{2}\right|_{\mathrm{H}}=\left|\gamma_{2}^{-1}\right|_{\mathrm{H}}=|\eta|$ and, moreover,

$$
\left|\gamma_{1} \gamma_{2}^{-1}\right|_{H}=\left|(\xi, \eta)_{\theta, \varphi}\right| \text {. }
$$


Thus, (2.2) and (2.3) imply the right-hand side of (2.1) and

$$
\left|\gamma_{1}\right|_{\mathrm{H}}=\left|\gamma_{1} \gamma_{2}^{-1} \gamma_{2}\right|_{\mathrm{H}} \leq\left|\gamma_{1} \gamma_{2}^{-1}\right|_{\mathrm{H}}+\left|\gamma_{2}\right|_{\mathrm{H}}
$$

gives the left-hand side. The proof is finished.

Lemma 2.2. Let $\psi$ be a positive function on $X$ supported on $\{\xi \in X:|\xi| \leq 1\}$ with $\int_{X} \psi d \mu=1$. Then,

$$
\tau-1 \leq \psi * \tau \leq \tau+1 .
$$

Proof. By using (2.1) and the definition (1.3) we get

$$
\tau(\xi)-\tau(\eta) \leq T^{\eta} \tau(\xi) \leq \tau(\xi)+\tau(\eta) .
$$

So

$$
\begin{aligned}
\psi * \tau(\xi) & =\int_{X} \psi(\eta) T^{\eta} \tau(\xi) d \mu(\eta) \\
& \leq \tau(\xi) \int_{|\eta| \leq 1} \psi(\eta) d \mu(\eta)+\int_{|\eta| \leq 1} \psi(\eta) \tau(\eta) d \mu(\eta) \\
& \leq \tau(\xi)+1 .
\end{aligned}
$$

Similarly, we obtain $\psi * \tau(\xi) \geq \tau(\xi)-1$. This finishes the proof of Lemma 2.2.

Now, for any positive function $w$ on $X$ let $L^{1}(X, w d \mu)$ denote the space of functions $f$ on $X$ such that

$$
\|f\|_{w}=\|f\|_{L^{1}(w d \mu)}=\int_{X}|f(\xi)| w(\xi) d \mu(\xi)
$$

is finite. Next, for any $l \geq 0, \beta>0$ we set $w_{l}(\xi)=(1+|\xi|)^{l}$ and $e(\beta)(\xi)=$ $\exp (\beta|\xi|)$. With this notation we have

Lemma 2.3. $L^{1}\left(X, w_{l} d \mu\right)$ and $L^{1}(X, e(\beta) d \mu)$ equipped with product (1.4) are Banach algebras.

Proof. Suppose that a positive function $w$ satisfies

$$
T^{\bar{\eta}} w(\xi) \leq w(\eta) w(\xi), \quad \eta, \xi \in X
$$

(recall that $\bar{\eta}=(y,-u)$ for $\eta=(y, u))$. Then, for any pair of positive functions $f, g$ on $X$

$$
\begin{aligned}
\|f * g\|_{w} & =\int_{X} \int_{X} f(\eta) T^{\eta} g(\xi) d \mu(\eta) w(\xi) d \mu(\xi) \\
& =\int_{X} f(\eta) \int_{X} g(\xi) T^{\bar{\eta}} w(\xi) d \mu(\xi) d \mu(\eta) \\
& \leq\|f\|_{w}\|g\|_{w} .
\end{aligned}
$$

Therefore, to prove Lemma 2.3 it suffices to show (2.5) for $w=w_{l}$ and $w=$ $e(\beta)$. Note, that since

$$
\left|(\xi, \bar{\eta})_{\theta, \varphi}\right| \leq|\xi|+|\eta|, \quad \xi, \eta \in X
$$


then also

$$
\left(1+\left|(\xi, \eta)_{\theta, \varphi}\right|\right)^{l} \leq(1+|\xi|)^{l}(1+|\eta|)^{l} .
$$

Thus, integrating (2.6) with respect to the probability measure $d \nu(\theta, \varphi)$ we obtain (2.5) for $w=w_{l}$. The case $w=e(\beta)$ follows the same way and the proof is completed.

Consider now an arbitrary commutative Banach $*$-algebra $B$ and a hermitian element $f=f^{*} \in B$. A function $F: \mathbb{R} \rightarrow \mathbb{C}$ is said to operate on $f$ provided the spectrum $\sigma(f)$ is contained in $\mathbb{R}$ and there is a $g \in B$ such that $F \circ \hat{f}=$ $\hat{g}$. Here $\hat{f}$ and $\hat{g}$ denote the Gelfand transforms of $f$ and $g$, respectively. Though we do not assume that $B$ contains the identity, we use the notation $e^{h}-1, h \in B$, to denote the element of $B$ defined by $e^{h}-1=\sum_{k=1}^{\infty}(1 / k) ! h^{k}$. There is the following classic criterion for a function $F$ to operate on $f \in B$.

Theorem 2.4 [6]. If $\left\|e^{i n f}-1\right\|_{B} \leq C n^{a}$, then $\sigma(f) \subseteq \mathbb{R}$ and every $F \in \mathrm{C}^{N}(\mathbb{R})$, $N>a+1$, with $F(0)=0$, operates on $f$.

We shall apply this criterion in $\S 4$ considering the algebras $L^{1}\left(X, w_{l} d \mu\right)$, $l \geq 0$. Now, we prove the following result.

Lemma 2.5. Suppose $f=f^{*} \in L^{2}(\mu)$ and $\|f\|_{e(\beta)}<\infty$ for all $0<\beta<\beta_{0}, \beta_{0}$ being a real number. Then, for any $l \geq 0$

$$
\left\|e^{i n f}-1\right\|_{w_{l}} \leq C n^{(\alpha+2+l} \text {. }
$$

Proof. By (1.18)

$$
\mu\{|\xi|<m\}=C_{1} m^{2 \alpha+2}, \quad m>0,
$$

with a constant $C_{1}>0$. Fix $\beta<\beta_{1}<\beta_{0}$. Then

$$
(1+|\xi|)^{l} \exp (\beta|\xi|) \leq C \exp \left(\beta_{1}|\xi|\right)
$$

and, by Lemma 2.3 ,

$$
\left\|e^{i n f}-1\right\|_{e\left(\beta_{1}\right)} \leq \exp \left(\|n f\|_{e\left(\beta_{1}\right)}\right) .
$$

Thus, for any $m>0$,

$$
\begin{aligned}
\left\|e^{i n f}-1\right\|_{w_{l}} \leq & \int_{|\xi|<m}\left|\left(e^{i n f}-1\right)(\xi)\right| w_{l}(\xi) d \mu(\xi) \\
& +\int_{|\xi| \geq m}\left|\left(e^{i n f}-1\right)(\xi)\right| \exp (\beta|\xi|) w_{l}(\xi) \exp (-\beta|\xi|) d \mu(\xi),
\end{aligned}
$$

and, assuming for a moment that $\left\|e^{i n f}-1\right\|_{2} \leq n\|f\|_{2}$, by Schwarz' inequality, (2.8) and (2.9), we estimate

$$
\begin{aligned}
\left\|e^{i n f}-1\right\|_{\omega^{\prime},} \leq & (1+m)^{l} \mu\{|\xi|<m\}^{1 / 2}\left\|e^{i n f}-1\right\|_{2} \\
& +C \exp (-\beta m)\left\|e^{i n f}-1\right\|_{e\left(\beta_{1}\right)} \\
\leq & (1+m)^{l} m^{(\alpha+1} n\|f\|_{2}+C \exp \left(n\|f\|_{e\left(\beta_{1}\right)}-\beta m\right) .
\end{aligned}
$$


Now, for $m=n / \beta\|f\|_{e\left(\beta_{1}\right)}$, we obtain

$$
\begin{aligned}
\left\|e^{i n f}-1\right\|_{w_{l}} & \leq C_{1}\left(1+\frac{n}{\beta}\|f\|_{e\left(\beta_{1}\right)}\right)^{l}\left(\frac{n}{\beta}\|f\|_{e\left(\beta_{1}\right)}\right)^{\alpha+1} n\|f\|_{2}+C \\
& \leq C n^{\alpha+l+2} .
\end{aligned}
$$

To prove the inequality $\left\|e^{i n f}-1\right\|_{2} \leq n\|f\|_{2}$, we note that $e^{\text {inf }}-1=T f$, where the operator $T$ on $L^{2}(\mu)$ is defined by

$$
T=\operatorname{inI}+\sum_{k=1}^{\infty} 1 /(k+1) !\left(\text { in } R_{f}\right)^{k}
$$

and $R_{f} g=f * g, g \in L^{2}(\mu)$. But $f=f^{*}$ so $T$ is a normal operator and therefore, by the spectral theorem,

$$
\|T\| \leq \sup \left\{\left|e^{i n t}-1\right||t|^{-1}: t \in \mathbb{R}\right\}=n .
$$

Thus the proof of Lemma 2.5 is completed.

\section{ESTIMATES FOR THE OPERATOR $L$}

First we prove two formulas, a Plancherel and an inversion formula, for the transform defined in $\S 1$. Let $\mathscr{M}=\mathbb{R} \times \mathbb{N}$ and $d \delta(\lambda, n)=\frac{1}{2 \pi}|\lambda|^{\alpha} d \lambda \oplus \gamma(n) d n$, where $\gamma(n)=(2 \Gamma(n+\alpha)) /\left(\Gamma(\alpha)^{2} n !\right)$. Here $\oplus$ denotes the sign of direct sum and $d n$ is the counting measure on $\mathbb{N}$. With such a notation we have:

Lemma 3.1. For every $f \in L^{1} \cap L^{2}(\mu)$ Plancherel's identity

$$
\|f(x, t)\|_{L^{2}(\mu)}=\|\hat{f}(\lambda, n)\|_{L^{2}(\mathscr{M}, d \delta)}
$$

holds. Moreover, for any reasonable function $f \in L^{1}(\mu)$ (for instance, for any $f \in \mathscr{D}(X))$, the inversion formula

$$
f(x, t)=\int_{\mathscr{M}} \hat{f}(\lambda, n) \varphi_{\lambda, n}(x,-t) d \delta(\lambda, n)
$$

is valid.

Proof. For the functions $\varphi_{\lambda, n}$ defined by (1.6) we write

$$
\varphi_{\lambda, n}(x, t)=\gamma(n)^{-1 / 2} e^{i \lambda t} \varphi_{n}\left(|\lambda|^{1 / 2} x\right),
$$

where $\varphi_{n}(x)=(2 n ! / \Gamma(n+\alpha))^{1 / 2} \exp \left(-x^{2} / 2\right) L_{n}^{\alpha-1}\left(x^{2}\right)$. Since the Laguerre functions $\mathscr{L}_{n}^{a}, n=0,1,2, \ldots$, form a complete orthonormal system in $L^{2}\left(\mathbb{R}_{+}, d x\right)$, the functions $\varphi_{n}, n=0,1,2, \ldots$, give a complete orthonormal basis in $L^{2}\left(\mathbb{R}_{+}, x^{2 \alpha-1} d x\right)$. Therefore, for any $g \in L^{1} \cap L^{2}\left(\mathbb{R}_{+}, x^{2 \alpha-1} d x\right)$ we have

$$
\|g\|_{L^{2}\left(x^{2 n-1} d x\right)}=\left(\sum_{n=0}^{\infty}|\hat{g}(n)|^{2}\right)^{1 / 2}
$$


where

$$
\hat{g}(n)=\int_{0}^{\infty} g(x) \varphi_{n}(x) x^{2 \alpha-1} d x .
$$

Consider now any function $f \in L^{1} \cap L^{2}(\mu)$ of the type $f(x, t)=g(x) h(t)$, with $g \in L^{1} \cap L^{2}\left(\mathbb{R}_{+}, x^{2 \alpha-1} d x\right), h \in L^{1} \cap L^{2}(\mathbb{R}, d t)$. Denote $g_{|\lambda|}(x)=$ $|\lambda|^{-\alpha} g\left(x /|\lambda|^{1 / 2}\right)$. Using (3.3) we write

$$
\begin{aligned}
\hat{f}(\lambda, n) & =\int_{\mathbb{R}} \int_{0}^{\infty} f(x, t) \varphi_{\lambda, n}(x, t) x^{2 \alpha-1} d x d t \\
& =\gamma(n)^{-1 / 2} \int_{0}^{\infty} g(x) \varphi_{n}\left(|\lambda|^{1 / 2} x\right) x^{2 \alpha-1} d x \int_{\mathbb{R}} h(t) e^{i \lambda t} d t \\
& =\gamma(n)^{-1 / 2} \int_{0}^{\infty} g_{|\lambda|}(x) \varphi_{n}(x) x^{2 \alpha-1} d x \hat{h}(-\lambda) \\
& =\gamma(n)^{-1 / 2}\left(g_{|\lambda|}\right)^{-}(n) \hat{h}(-\lambda) .
\end{aligned}
$$

Here we abuse slightly the notation using the same symbol ${ }^{-}$to denote three different transforms: $\hat{f}(\lambda, n)$ is defined by (1.8), $\hat{h}$ denotes the ordinary Fourier transform of $h \in L^{1} \cap L^{2}(\mathbb{R})$ and, eventually, $\left(g_{|\lambda|}\right)^{\wedge}$ is the transform of the function $g_{|\lambda|} \in L^{1} \cap L^{2}\left(x^{2 \alpha-1} d x\right)$ with respect to the orthogonal basis $\varphi_{n}$, $n=0,1,2, \ldots$, (cf. (3.5)). Now, since $|\lambda|^{\alpha / 2}\left\|g_{|\lambda|}\right\|_{L^{2}\left(x^{2 n-1} d x\right)}=\|g\|_{L^{2}\left(x^{2 n-1} d x\right)}$, using (3.4) and Plancherel's theorem for the ordinary Fourier transform, we get

$$
\begin{aligned}
\|\hat{f}(\lambda, n)\|_{L^{2}(\mathscr{K}, \delta)}^{2} & =\sum_{n=0}^{\infty} \gamma(n) \frac{1}{2 \pi} \int_{\mathbb{R}}|\hat{f}(\lambda, n)|^{2}|\lambda|^{\alpha} d \lambda \\
& =\frac{1}{2 \pi} \int_{\mathbb{R}}|\hat{h}(-\lambda)|^{2}\left(\sum_{n=1}^{\infty}\left|\hat{g}_{|\lambda|}(n)\right|^{2}\right)|\lambda|^{\alpha} d \lambda \\
& =\|g\|_{L^{2}\left(x^{2 n-1} d x\right)}^{2}\|h\|_{L^{2}(\mathbb{R})}^{2}=\|f(x, t)\|_{2}^{2} .
\end{aligned}
$$

This completes the proof of (3.1) for any function $f(x, t)$ with separate variables.

A standard limiting argument proves (3.1) for any $f \in L^{1} \cap L^{2}(\mu)$. The proof of (3.2) follows the same pattern.

We are now in a position to prove a Sobolev-type inequality for the operator $L$.

Theorem 3.2. If $M>(\alpha+1) / 2$ is a positive integer then

$$
\|f\|_{\infty} \leq C\left\|(I+L)^{M} f\right\|_{2}
$$

for any reasonable function $f \in L^{1}(\mu)$ (for instance for any $f \in \mathscr{D}(X)$ ).

Proof. Let $f$ be a suitable function on $X$ and $M>(\alpha+1) / 2$ be a positive integer. Using (1.11) and the inversion formula (3.2), we write

$$
f(x, t)=\int_{\mathscr{M}}(1+4|\lambda|(\alpha / 2+n))^{-M}\left((I+L)^{M} f\right)^{\wedge}(\lambda, n) \varphi_{\lambda, n}(x,-t) d \delta(\lambda, n) .
$$


Consequently, applying Schwarz' inequality and Plancherel's identity (3.1), we get (recall that $\left|\varphi_{\lambda, n}\right| \leq 1$ )

$$
\|f\|_{\infty} \leq\left\|(1+4|\lambda|(\alpha / 2+n))^{-M}\right\|_{L^{2}(M)}\left\|(I+L)^{M} f\right\|_{2} .
$$

It suffices now only to prove that

$$
\left\|(1+4|\lambda|(\alpha / 2+n))^{-M}\right\|_{L^{2}(\mathscr{M})}<\infty .
$$

We have

$$
\begin{aligned}
\left\|(1+4|\lambda|(\alpha / 2+n))^{-M}\right\|_{L^{2}(\mathscr{M})}^{2} \\
=\sum_{n=0}^{\infty} \gamma(n) \frac{1}{2 \pi} \int_{\mathbb{R}}(1+4|\lambda|(\alpha / 2+n))^{-2 M}|\lambda|^{\alpha} d \lambda \\
=\frac{1}{\pi \Gamma(\alpha)^{2}} \sum_{n=0}^{\infty} \Gamma(n+\alpha) / n ! \cdot I_{M, n},
\end{aligned}
$$

where by $I_{M, n}$ we denote the last integral over $\mathbb{R}$. Since $2 M>\alpha+1$ we estimate $I_{M, n} \leq C \int_{0}^{\infty}(1+\lambda n)^{-2 M} \lambda^{\alpha} d \lambda=C_{1} n^{-\alpha-1}$. But $\Gamma(n+\alpha) / n ! \sim n^{\alpha-1}$ as $n \rightarrow \infty$ so, eventually,

$$
\sum_{n=1}^{\infty} \frac{\Gamma(n+\alpha)}{n !} I_{M, n} \leq C \sum_{n=1}^{\infty} n^{-2}<\infty .
$$

This completes the proof of (3.7) and hence the theorem. The following lemma contains three estimates, which will be crucial in the proof of Theorem 4.1.

Lemma 3.3. Let $\psi \in \mathscr{D}(X)$ be a positive function supported on $\{\xi \in X:|\xi| \leq 1\}$ with $\int_{X} \psi d \mu=1$. Then

$$
\begin{gathered}
\|L(\psi * \tau)\|_{\infty} \leq C, \\
\left\|x \partial_{t}(\psi * \tau)\right\|_{\infty} \leq C, \\
\left\|\partial_{x}(\psi * \tau)\right\|_{\infty} \leq C .
\end{gathered}
$$

Proof. First, we show (3.8). Since

$$
|L(\psi * \tau)|=|\psi * L \tau| \leq \psi *|L \tau|,
$$

and $\psi * \tau$ as well as $L(\psi * \tau)$ are continuous on the closure of $X$ in $\mathbb{R}^{2}$, so (3.8) amounts to showing that

$$
(\psi *|L \tau|)(\xi) \leq C
$$

on $|\xi| \geq 2$. Straightforward calculation yields

$$
\begin{aligned}
\partial_{x} \tau(x, t) & =x^{3} /|(x, t)|^{3}, \\
\partial_{x x} \tau(x, t) & =3 x^{2}\left(|(x, t)|^{4}-x^{4}\right) /|(x, t)|^{7}, \\
\partial_{t} \tau(x, t) & =2 t /|(x, t)|^{3}, \\
\partial_{t t} \tau(x, t) & =2\left(|(x, t)|^{4}-6 t^{2}\right) /|(x, t)|^{7},
\end{aligned}
$$


and the above gives $L \tau(x, t)=(2 \alpha+1) x^{2} \tau(x, t)^{-3}$. Therefore $|L \tau(x, t)| \leq$ $C /|(x, t)|$. Now, for $|\xi| \geq 2,|\eta| \leq 1$, by (2.1) we get

$$
\left|(\xi, \eta)_{\theta, \varphi}\right| \geq|\xi|-|\eta| \geq|\xi| / 2
$$

and, consequently,

$$
T^{\eta}(|L \tau|)(\xi) \leq C \int_{0}^{\infty} \int_{0}^{\infty} 1 /\left|(\xi, \eta)_{\theta, \varphi}\right| d \nu(\theta, \varphi) \leq C /|\xi| .
$$

Eventually, for $|\xi| \geq 2$,

$$
\psi *|L \tau|(\xi)=\int_{|\eta| \leq 1} \psi(\eta) T^{\eta}(|L \tau|) d \mu(\eta) \leq C /|\xi| .
$$

This gives (3.11) and finishes the proof of (3.8). As far as (3.9) is concerned, since $\partial / \partial t$ also commutes with the convolution, i.e., we have

$$
\partial_{t}(\psi * \tau)=\psi *\left(\partial_{t} \tau\right)
$$

we may repeat all arguments used above (now only (3.14) is needed) to get the required estimate (3.9). To finish the proof of Lemma 3.3, i.e., to verify (3.10), we note that with the notation $A(x)=x^{2 x-1}$ we have

$$
\partial_{x} g(x, t)=\frac{1}{A(x)} \int_{0}^{x}\left(L-y^{2} \partial_{t t}\right) g(y, t) A(y) d y .
$$

Suppose first that $|t| \geq 2$. Then $|(y, t)| \geq 2,1 /|(y, t)| \leq 1 / y$, and (3.16) gives

$$
\frac{1}{A(x)} \int_{0}^{x}|L(\psi * \tau)(y, t)| A(y) d y \leq C .
$$

Therefore it remains to show that

$$
\left|\partial_{t t}(\psi * \tau)(\xi)\right| \leq C /|\xi|^{3},
$$

which immediately implies

$$
\frac{1}{A(x)} \int_{0}^{x} y^{2}\left|\partial_{t t}(\psi * \tau)(y, t)\right| A(y) d y \leq C .
$$

Thus, in virtue of (3.17), combining (3.18) and (3.19) gives (3.10). We remark that $(3.19)$ is a consequence of $(3.15)$, since $\partial^{2} / \partial t^{2}$ also commutes with the convolution and it suffices to repeat once more all arguments used in the proof of (3.11).

Now, let $|t| \leq 2$. Then (3.16) and (3.19) remain still valid for $x \geq 2$ (then $|\xi|=|(x, t)| \geq 2)$ and, as before, imply (3.18) and (3.19). The proof of Lemma 3.3 is completed.

\section{A functional Calculus}

Our aim in this section is to construct a functional calculus for the operator $L$. This is done in Theorem 4.3, the main result of this section. We start by showing that the heat kernel $\left(p_{u}\right)_{u>0}$ associated to $L$, in some sense, rapidly decreases at infinity. More precisely we prove the following: 
Theorem 4.1. There exist positive constants $A$ and $C$ such that for all $s \geq 1$

$$
\int_{X} p_{1}(\xi) \exp (s|\xi|) d \mu(\xi) \leq C \exp \left(A s^{2}\right)
$$

Note, that $(4.1)$ then implies

$$
\int_{X} p_{1}(\xi) \exp \left(-A\left(2 s-\frac{|\xi|}{4 A}\right)^{2}\right) \exp \left(\frac{|\xi|^{2}}{16 A}\right) d \mu(\xi) \leq C \exp \left(-3 A s^{2}\right)
$$

and integrating over $\mathbb{R}$ with respect to $s$ (clearly (4.1) holds also for $s<1$ ) we get an immediate

Corollary 4.2. There is an $\varepsilon>0$ (for instance, $\varepsilon=\frac{1}{16 A}$ ) such that

$$
\int_{X} p_{1}(\xi) \exp \left(\varepsilon|\xi|^{2}\right) d \mu(\xi)<\infty .
$$

Proof of Theorem 4.1. We fix a positive $C^{\infty}$-function $\psi \in \mathscr{D}(X)$ supported on $\{\xi \in X:|\xi| \leq 1\}, \int_{X} \psi d \mu=1$. Then, for any $s \geq 1$ we denote $\psi^{(s)}(\xi)=$ $\exp (s(\psi * \tau(\xi)+1))$. Straightforward calculation yields

$$
L \psi^{(s)}=\left(s L(\psi * \tau)+s^{2}\left(\partial_{x}(\psi * \tau)\right)^{2}+s^{2}\left(x \cdot \partial_{t}(\psi * \tau)\right)^{2}\right) \psi^{(s)} .
$$

Therefore, using the estimates contained in Lemma 3.3, we get

$$
\left|L \psi^{(s)}(\xi)\right| \leq A s^{2} \psi^{(s)}(\xi),
$$

with a constant $A>0$ independent of $\xi \in X$ and $s \geq 1$. Recall now, that $\rho_{1 / k} \psi, k=1,2, \ldots$, is an approximate identity in $L^{\overline{1}}(\mu)$, which means, in particular, that

$$
p_{u} * \rho_{1 / k} \psi \rightarrow p_{u}
$$

near uniformly as $k \rightarrow \infty$ (for any $u>0$ ). Let $\langle$,$\rangle denote the inner product$ in $L^{2}(\mu)$. For any $s \geq 1$, and $k \in \mathbb{N}$ define $f_{s, k}(u)=\left\langle p_{u} * \rho_{1 / k} \psi, \psi^{(s)}\right\rangle$. Then, using (1.14) and (4.3), we get

$$
\begin{aligned}
\left|d / d u f_{s, k}(u)\right| & =\left|\left\langle\partial / \partial u\left(p_{u} * \rho_{1 / k} \psi\right), \psi^{(s)}\right\rangle\right| \\
& =\left|\left\langle p_{u} * \rho_{1 / k} \psi, L \psi^{(s)}\right\rangle\right| \\
& \leq A s^{2}\left\langle p_{u} * \rho_{1 / k} \psi, \psi^{(s)}\right\rangle \\
& \leq A s^{2} f_{s, k}(u) .
\end{aligned}
$$

That means that the positive function $f_{s, k}(u)$ satisfies

$$
\left|(d / d u) \ln f_{s, k}(u)\right| \leq A s^{2},
$$

with a constant $A$ independent of $k \in \mathbb{N}$, and, next, (4.5) implies $f_{s, k}(u) \leq$ $C \exp \left(A s^{2} u\right)$. In particular, for $u=1$ we get $\left\langle p_{1} * \rho_{1 / k} \psi, \psi^{(s)}\right\rangle \leq C \exp \left(A s^{2}\right)$, 
with constants $A$ and $C$ independent of $k \in \mathbb{N}$. Thus, using (4.4), we obtain $\left\langle p_{1}, \psi^{(s)}\right\rangle \leq C \exp \left(A s^{2}\right)$ and, eventually, by $(2.4)$,

$$
\int_{X} p_{1}(\xi) \exp (s \tau(\xi)) d \mu(\xi) \leq\left\langle p_{1}, \psi^{(s)}\right\rangle \leq C \exp \left(A s^{2}\right) .
$$

This finishes the proof of Theorem 4.1 .

We are now in a position to state our main result, which has been inspired by Theorem 1.12 of [5]. For any $l \geq 0$, let $\mathbb{A}(l)$ denote the smallest closed subalgebra in $L^{1}(X, w, d \mu)$ generated by the set $\left\{p_{u}: u>0\right\}$. By $\hat{f}$ denote the Gelfand transform of an element $f \in \mathbb{A}(l)$. To be precise we should write $\hat{f}^{\mathrm{A}(l)}$ instead of $\hat{f}$ but, for simplicity, we abuse the notation slightly. Clearly, for any $l \geq 0$, the Gelfand space of $\mathbb{A}(l)$ is homeomorphic to $\mathbb{R}_{+}$and one has

$$
\left(p_{u}\right)^{\wedge}(\lambda)=\exp (-u \lambda), \quad \lambda \geq 0
$$

Our result then is

Theorem 4.3. Let $l \geq 0$ be a fixed real number and $N>l+\alpha+3, M>(\alpha+1) / 2$ be positive integers. Then, for any $K \in C^{N}\left(\mathbb{R}_{+}\right)$satisfying the condition

$$
\sup _{\lambda>0}\left|K^{(j)}(\lambda)\right|(1+\lambda)^{M N+1}<\infty, \quad j=0,1,2, \ldots, N,
$$

there is an element $k \in \mathbb{A}(l)$ with $\hat{k}=K$.

Proof. We will operate, in sense of the definition preceding Theorem 2.4, on the element $R_{1}^{* M} \in \mathbb{A}(l)$, the $M$ th convolution power of $R_{1} \in \mathbb{A}(l)$ defined by

$$
R_{1}=\int_{0}^{\infty} e^{-u} p_{u} d u
$$

First, note that $\left(R_{1}^{* M}\right)^{\wedge}(\lambda)=(1+\lambda)^{-M}$. Further, using the Sobolev-type inequality (3.6), for any $\varphi \in \mathscr{D}(X)$ we get

$$
\begin{aligned}
\left|\left\langle R_{1}^{* M}, \varphi\right\rangle\right| & =\left|R_{1}^{* M} * \varphi(0,0)\right| \\
& \leq C\left\|(I+L)^{M}\left(R_{1}^{* M} * \varphi\right)\right\|_{2} \\
& \leq C\|\varphi\|_{2},
\end{aligned}
$$

which proves that $R_{1}^{* M} \in L^{2}(\mu)$. Next, we show that $\left\|R_{1}\right\|_{e(\beta)}<\infty$, hence also $\left\|R_{1}^{* M}\right\|_{e(\beta)}<\infty$, for any $\beta, 0<\beta<\beta_{0}, \beta_{0}$ being a positive constant. Since 
$p_{u}=\rho_{u^{1 / 2}} p_{1}$ we obtain

$$
\begin{aligned}
\left\|R_{1}\right\|_{e(\beta)} & =\int_{X} R_{1}(\xi) \exp (\beta|\xi|) d \mu(\xi) \\
& =\int_{0}^{\infty} e^{-u} \int_{X} \rho_{u^{1 / 2}} p_{1}(\xi) \exp (\beta|\xi|) d \mu(\xi) d u \\
& =\int_{0}^{\infty} e^{-u} \int_{X} p_{1}(\xi) \exp \left(\beta u^{1 / 2}|\xi|\right) d \mu(\xi) d u \\
& =\int_{X} p_{1}(\xi) \int_{0}^{\infty} \exp \left(-u+\beta u^{1 / 2}|\xi|\right) d u d \mu(\xi) \\
& \leq \int_{X} p_{1}(\xi) \exp \left(\beta^{2}|\xi|^{2} / 4\right) d \mu(\xi) \\
& <\infty,
\end{aligned}
$$

provided that $\beta_{0}^{2} / 4<\varepsilon$, where $\varepsilon$ is as in Corollary 4.2. Now, to prove the theorem, consider a function $K$ that satisfies (4.6) and define $F(\lambda)=K\left(\lambda^{-1 / M}-1\right)$ when $\lambda>0$ and 0 otherwise. Then $F \in C^{N}(\mathbb{R}), F(0)=0$. Furthermore, $f=R_{1}^{* M}$ satisfies the assumptions of Lemma 2.5 and hence we may use Theorem 2.4 to conclude that $F$ operates on $R_{1}^{* M}$. This means that $K=F \circ\left(R_{1}^{* M}\right)^{\wedge}=\hat{k}$, for some $k \in \mathbb{A}(l)$. The theorem is proved.

The positive symmetric in $L^{2}(\mu)$ operator $L$ being hypoelliptic admits a selfadjoint extension. Denote it by $\bar{L}$. Let $E_{\lambda}$ be the spectral resolution for $\bar{L}$, i.e.,

$$
\bar{L} f=\int_{0}^{\infty} \lambda d E_{\lambda}(f), \quad f \in \operatorname{Dom}(\bar{L}) .
$$

Now, as an immediate consequence of Theorem 4.3 we obtain

Corollary 4.4. Let a function $K$ on $\mathbb{R}_{+}, K(0)=1$, satisfy the assumptions (4.6) with $l=0$. Then the operator

$$
T_{K_{t}} f=\int_{0}^{\infty} K(t \lambda) d E_{\lambda}(f)
$$

is bounded on all $L^{p}(\mu), 1 \leq p<\infty$, and, moreover,

$$
\lim _{t \rightarrow 0}\left\|f-T_{K_{t}} f\right\|_{p}=0, \quad f \in L^{p}(\mu) .
$$

Proof. By Theorem 4.3 $T_{K} f=k * f$ with a kernel $k \in L^{1}(\mu)$. Hence $T_{K}$ is bounded on $L^{p}(\mu), 1 \leq p<\infty$. Moreover, $K(0)=1$ implies $\int_{X} k d \mu=1$. Further, $T_{K_{t}} f=\rho_{t^{1 / 2}} k * f$ and (4.7) is a consequence of the fact that $\rho_{t^{1 / 2}} k$ is an approximate identity as $t \rightarrow 0$.

Remark 4.5. After the paper was submitted for publication, the author observed that the estimate contained in Corollary 4.2 may be also obtained via recent results of D. Jerison and A. Sánchez-Calle. In [10] they established pointwise estimates for the heat kernels of a large class of subelliptic differential operators. 
More precisely, they considered second order partial differential operators of the form

$$
L=\sum_{i, j=1}^{n} \frac{1}{h(y)} \frac{\partial}{\partial y_{i}}\left(h(y) a_{i j}(y) \frac{\partial}{\partial y_{j}}\right)
$$

defined on an open set $\bar{\Omega}$ of $\mathbb{R}^{n}$, where the coefficients $a_{i j}$ and $h$ are $C^{\infty}$ real-valued functions on $\bar{\Omega}, h$ is positive, and the matrix $A(y)=\left(a_{i j}(y)\right)$ is symmetric positive semidefinite for every $y \in \bar{\Omega}$. Moreover, $L$ is assumed to satisfy a subelliptic estimate. With this assumption or $L$, D. Jerison and A. Sánchez-Calle proved $[10, \mathrm{p} .60]$ that the heat kernel $h\left(t, y_{1}, y_{2}\right)$ associated to $L$ satisfies

$$
h\left(t, y_{1}, y_{2}\right) \leq A \mu\left(B\left(y_{1}, t^{1 / 2}\right)\right)^{-1} e^{-d\left(y_{1}, y_{2}\right)^{2} / y t},
$$

for some positive constants $A, \gamma$. Here $d=d_{L}$ is a distance function naturally associated to $L, B\left(y_{1}, t^{1 / 2}\right)$ denotes the ball in this metric and $d \mu(y)=$ $h(y) d y$.

In our context $\Omega=\left\{y \in \mathbb{R}^{2}: y=(x, t), x>0\right\}$ and the operator $-L$ we consider is of the form (4.8) with $h(x, t)=x^{2 x-1}$ and

$$
A(x, t)=\left(\begin{array}{cc}
1 & 0 \\
0 & x^{2}
\end{array}\right) \text {. }
$$

Furthermore, $-L$ satisfies the required subelliptic estimate since it can be written as $-L=Y_{1}^{2}+Y_{2}^{2}+Y_{0}$, with

$$
Y_{1}=\frac{\partial}{\partial x}, \quad Y_{2}=x \frac{\partial}{\partial x}, \quad Y_{0}=\frac{2 \alpha-1}{x} \frac{\partial}{\partial x} .
$$

It is also not hard to prove that then (4.9) becomes

$$
p_{1}(\xi) \leq C \exp \left(-\eta|\xi|^{2}\right),
$$

with some positive constants $\eta$ and $C$. This clearly implies (4.2).

\section{MEAN CONVERGENCE OF SOME SUMMABILITY METHODS FOR LAGUERRE SERIES}

In this section we apply the functional calculus described in Theorem 4.3 to get mean convergence results for Laguerre series.

First, we define a periodic analogy of the convolution structure discussed in $\S 2$. By $(\tilde{X}, \tilde{\mu})$ we denote the measure space $\tilde{X}=\mathbb{R}_{+} \times(0,2 \pi)$ equipped with the measure $d \tilde{\mu}(x, t)=x^{2(x-1} d x d t, d t$ being the Lebesgue measure on $(0,2 \pi)$. We identify functions on $\widetilde{X}$ with $2 \pi$-periodic functions on $X$ with respect to the $t$-variable. By $\widetilde{L}$ we denote the operator $L$ acting on functions on $\widetilde{X}$. The convolution on $L^{1}(\widetilde{X}, \tilde{\mu})$ is now defined by

$$
f * g(\xi)=\int_{\widetilde{X}} T^{\eta} f(\xi) g(\eta) d \mu(\eta)
$$


$f, g \in L^{1}(\tilde{X}, \tilde{\mu}), \xi \in \widetilde{X}$, where the generalized translations $T^{\eta}, \eta \in \tilde{X}$, are given, as before, by (1.3).

As just remarked, we abuse the notation slightly (and we will do so in the sequel) using symbols like ${ }^{*}, T^{\eta}$, and - to denote different objects connected with $(X, \mu)$ and the periodic case $(\tilde{X}, \tilde{\mu})$ on the other hand.

For any function $f$ on $X$ we write

$$
\dot{f}(x, t)=\sum_{k=-\infty}^{\infty} f(x, t+2 k \pi) .
$$

As one can easily verify the correspondence $f \rightarrow \dot{f}$ maps $L^{p}(X, \mu)$ onto $L^{p}(\tilde{X}, \tilde{\mu}), 1 \leq p<\infty$, and it is a linear contraction. Moreover, $(f * g)^{\circ}=\dot{f} * \dot{g}$ for any pair of functions $f, g$ for which $f * g$ makes sense (for instance, for $\left.f, g \in L^{1}(X, \mu)\right)$. It is clear that among the functions $\varphi_{\lambda, n}, \lambda \neq 0$, $n=0,1,2, \ldots$, defined by (1.6), only those are $2 \pi$-periodic for which $|\lambda| \in \mathbb{N}$. Thus the functions $\varphi_{m, n}, m= \pm 1, \pm 2, \ldots, n=0,1,2, \ldots$, are eigenfunctions for $\widetilde{L}$ and hence, for any $f \in L^{1}(\tilde{X}, \tilde{\mu})$, we define its transform $\hat{f}(m, n)$ by $\hat{f}(m, n)=\int_{\tilde{X}} f \varphi_{m, n} d \tilde{\mu}$. As one can check, for any $f \in L^{1}(X, \mu)$ we have

$$
(\dot{f})^{\wedge}(m, n)=\hat{f}(m, n) \text {. }
$$

Moreover, for any $f, g \in L^{1}(\tilde{X}, \tilde{\mu})$,

$$
(f * g) \wedge(m, n)=\hat{f}(m, n) \hat{g}(m, n) .
$$

Let $L_{0}^{p}(\tilde{X}, \tilde{\mu})$ denote the Banach subspace of these functions in $L^{p}(\tilde{X}, \tilde{\mu})$, which are of the form

$$
F(x, t)=e^{i t} f(x) .
$$

The correspondence $F \rightarrow f$ establishes an isometric isomorphism, up to the constant $(2 \pi)^{1 / p}$, between the spaces $L_{0}^{p}(\tilde{X}, \tilde{\mu})$ and $L^{p}\left(\mathbb{R}_{+}, x^{2 \alpha-1} d x\right)$. Straightforward calculation shows that $\widetilde{L} F$ is of the form (5.3) provided that $F$ does, so the functions

$$
\phi_{n}=(\gamma(n) /(2 \pi))^{1 / 2} \varphi_{1, n},
$$

$n=0,1,2, \ldots$, being elements of $L_{0}^{p}(\tilde{X}, \tilde{\mu})$ and forming an orthonormal basis in $L_{0}^{2}(\widetilde{X}, \tilde{\mu})$, are eigenfunctions of $\widetilde{L}$ acting in this space. From (3.3) one gets

$$
(2 \pi)^{1 / 2} \phi_{n}(x, t)=e^{i t} \varphi_{n}(x) .
$$

We have already observed that the functions $\varphi_{n}, n=0,1,2, \ldots$, form a complete orthonormal basis in $L^{2}\left(x^{2 \alpha-1} d x\right)$. Now, for any $F \in L_{0}^{p}(\tilde{X}, \tilde{\mu})$ being of the form (5.3) we have

$$
\left\langle F, \phi_{n}\right\rangle_{L_{0}^{2}(\widetilde{X}, \tilde{\mu})}=\int_{\widetilde{X}} F \bar{\phi}_{n} d \tilde{\mu}=(\gamma(n) /(2 \pi))^{1 / 2} \widehat{F}(-1, n)
$$


and (cf. (3.5))

$$
\begin{aligned}
\left\langle F, \phi_{n}\right\rangle_{L_{0}^{2}(\tilde{X}, \tilde{\mu})} & =(2 \pi)^{1 / 2} \int_{0}^{\infty} f(x) \varphi_{n}(x) x^{2(x-1} d x \\
& =(2 \pi)^{1 / 2} \hat{f}(n) .
\end{aligned}
$$

We are now ready to prove our main result. Let $L_{n}^{a}$ and $l_{n}^{a}, n=0,1,2, \ldots$, be the Laguerre polynomials and the functions given by $(0.3)$ respectively. The system $l_{n}^{a}, n=0,1,2, \ldots$, is an orthonormal basis in $L^{2}\left(\mathbb{R}_{+}, x^{a} d x\right)$ and for any function $f \in L^{p}\left(\mathbb{R}_{+}, x^{a} d x\right), 1 \leq p \leq \infty$, we write its formal Laguerre expansion $f \sim \sum_{n=0}^{\infty}\left(f, l_{n}^{a}\right) l_{n}^{a}$, where $\left(f, l_{n}^{a}\right)=\int_{0}^{\infty} f(x) l_{n}^{a}(x) x^{a} d x$. For any $a>0, n \in \mathbb{N}$, denote $\lambda_{n, a}=4(n+(a+1) / 2)$. Our result then is

Theorem 5.1. Let $a>0$ be a positive real number and $N>a+4, M>(a+2) / 2$ be positive integers. Suppose also that $K \in C^{N}\left(\mathbb{R}_{+}\right), K(0)=1$, satisfies the condition

$$
\sup _{\lambda \geq 0}\left|K^{(j)}(\lambda)\right|(1+\lambda)^{M N+1}<\infty, \quad j=0,1,2, \ldots, N .
$$

Then, for every $f \in L^{p}\left(x^{a} d x\right), 1 \leq p<\infty$, we have

$$
\lim _{t \rightarrow 0}\left\|f-\sum_{n=0}^{\infty} K\left(t \lambda_{n, a}\right)\left(f, l_{n}^{a}\right) l_{n}^{a}\right\|_{L^{p}\left(x^{a} d x\right)}=0 .
$$

Proof. We work with $\alpha>1$ and prove (5.7) with $a$ replaced with $\alpha-1$. By Theorem 4.3 there is a $k \in \mathbb{A}(0)$ such that $\hat{k}=K$. Further, $K(0)=1$, hence $\int_{X} k(x) d \mu(x)=1$. Since $\rho_{t^{1 / 2}} k$ is an approximate identity in $L^{p}(X, \mu)$ as $t \rightarrow 0$, thus, for any $g \in L^{p}(X, \mu)$ we get

$$
\lim _{t \rightarrow 0}\left\|g-\rho_{t^{1 / 2}} k * g\right\|_{L^{p}(X, \mu)}=0,
$$

which implies

$$
\lim _{t \rightarrow 0}\left\|\dot{g}-\left(\rho_{t^{1 / 2}} k * g\right)^{\cdot}\right\|_{L^{p}(\widetilde{X}, \tilde{\mu})}=0 .
$$

Hence

$$
\lim _{t \rightarrow 0}\left\|F-\left(\rho_{t^{1 / 2}} k\right)^{\cdot} * F\right\|_{L^{p}(\tilde{X}, \tilde{\mu})}=0,
$$

for any $F \in L_{0}^{p}(\tilde{X}, \tilde{\mu})$, since $L^{p}(X, \mu) \ni g \rightarrow \dot{g} \in L^{p}(\tilde{X}, \tilde{\mu})$ is onto. Note that $\hat{p}_{u}(-1, n)=\hat{p}_{u}\left(\lambda_{n, a-1}\right)$ for any $u>0$; hence also $\hat{k}(-1, n)=\hat{k}\left(\lambda_{n, a-1}\right)$ (here $\hat{k}(-1, n)$ is given by $(1.8)$ and $\hat{k}(s)$ is the Gelfand transform of $k \in \mathbb{A}(0))$. Furthermore, $\left(\rho_{t^{1 / 2}} k\right)^{\wedge}=K_{t}$ where $K_{t}(\lambda)=K(t \lambda)$.

Let $F \in L_{0}^{p}(\tilde{X}, \tilde{\mu})$ be given by (5.3). By writing the series expansion of $\left(\rho_{t^{1 / 2}} k\right)^{\circ} * F$ with respect to the system $\phi_{n}, n=0,1,2, \ldots$, (remark that $G * F \in L_{0}^{p}(\tilde{X}, \tilde{\mu})$ for any $\left.G \in L^{1}(\tilde{X}, \tilde{\mu})\right)$ and using (5.5), (5.2), and (5.1), 
we obtain

$$
\begin{aligned}
\left(\rho_{t^{1 / 2}} k\right)^{\cdot} * F & \sim \sum_{n=0}^{\infty}\left\langle\left(\rho_{t^{1 / 2}} k\right) * F, \phi_{n}\right\rangle_{L_{0}^{2}(\widetilde{x}, \tilde{\mu})} \phi_{n} \\
& =\sum_{n=0}^{\infty}\left(\rho_{t^{1 / 2}} k\right)^{-}(-1, n) \hat{f}(n)(2 \pi)^{1 / 2} \phi_{n} \\
& =e^{i t} \cdot \sum_{n=0}^{\infty} K\left(t \lambda_{n, \alpha-1}\right) \hat{f}(n) \varphi_{n} .
\end{aligned}
$$

Now, neglecting the factor $e^{i t}$, in virtue of (5.8) and (5.9) we obtain

$$
\lim _{t \rightarrow 0}\left\|f-\sum_{n=0}^{\infty} K\left(t \lambda_{n, \alpha-1}\right) \hat{f}(n) \varphi_{n}\right\|_{L^{p}\left(x^{2 x-1} d x\right)}=0
$$

for any $f \in L^{p}\left(x^{2 \alpha-1} d x\right), 1 \leq p<\infty$. To conclude, we remark only that for any $1 \leq p<\infty$ the correspondence $A f(x)=2^{-1 / 2} f\left(x^{1 / 2}\right)$ maps $L^{p}\left(x^{2 \alpha-1} d x\right)$ onto $L^{p}\left(x^{\alpha-1} d x\right)$ and

$$
\|A f\|_{L^{p}\left(x^{(x-1} d x\right)}=2^{1 / p-1 / 2}\|f\|_{L^{p}\left(x^{2(x-1} d x\right)} .
$$

Moreover, $A \varphi_{n}=l_{n}^{\alpha-1}, n=0,1,2, \ldots$, and

$$
\langle A f, A g\rangle_{L^{2}\left(x^{(x-1} d x\right)}=\langle f, g\rangle_{L^{2}\left(x^{2 x-1} d x\right)} .
$$

Therefore (5.10) implies (5.7) with $a$ replaced by $\alpha-1$ and the proof is completed.

We come now to the first application of Theorem 5.1, which deals with the classical Abel means. Since the function $K(\lambda)=\exp (-\lambda / 4)$ satisfies the assumptions of this theorem we easily obtain

Corollary 5.2. For any $f \in L^{p}\left(x^{a} d x\right), a>0,1 \leq p<\infty$,

$$
\lim _{r \rightarrow 1^{-}} \sum_{n=0}^{\infty} r^{n}\left(f, l_{n}^{a}\right) l_{n}^{a}=f,
$$

in $L^{p}\left(x^{a} d x\right)$.

The next application concerns the Bochner-Riesz means. If $N-1>a+4$, then the function $K(\lambda)=(1-\lambda / 4)^{N}, 0 \leq \lambda \leq 4$, and 0 otherwise, belongs to $C^{N-1}\left(\mathbb{R}_{+}\right)$and satisfies (5.6). Therefore, we easily get

Corollary 5.3. Let $N>a+5$. Then for any $f \in L^{p}\left(x^{a} d x\right)$

$$
\lim _{M \rightarrow \infty} \sum_{n=1}^{M}(1-n / M)^{N}\left(f, l_{n}^{a}\right) l_{n}^{a}=f
$$

in $L^{p}\left(x^{a} d x\right), 1 \leq p<\infty$. 


\section{REFERENCES}

1. R. Askey and S. Wainger, Mean convergence of expansions in Laguerre and Hermite series, Amer. J. Math. 87 (1965), 695-708.

2. J. Cygan, Subadditivity of homogeneous norms on certain nilpotent Lie groups, Proc. Amer. Math. Soc. 83 (1981), 69-70.

3. J. Dlugosz, Almost everywhere convergence of some summability methods for Laguerre series, Studia Math. 82 (1985), 199-209.

4. A. Hulanicki, Subalgebra of $L^{1}(G)$ associated with Laplacian on a Lie group, Coll. Math. 31 (1974), 259-287.

5. A. Hulanicki and J. Jenkins, Almost everywhere summability on nilmanifolds, Trans. Amer. Math. Soc. 278 (1983), 703-715.

6. Y. Katznelson, An introduction to harmonic analysis, Wiley, New York, 1968.

7. B. Muckenhoupt, Poisson integrals for Hermite and Laguerre expansions, Trans. Amer. Math. Soc. 139 (1969), 231-242.

8. K. Stempak, An algebra associated with the generalized sublaplacian, Studia Math. 88 (1988), 245-256.

9. __ A new proof of Watson's formula, Canad. Math. Bull. 31 (1988), 414-418.

10. D. Jerison and A. Sánchez-Calle, Subelliptic, second order differential operators, Lecture Notes in Math., vol. 1277, Springer-Verlag, 1987, pp. 46-77.

Institute of Mathematics, University of Wroclaw, Pl. Grunwaldzki $2 / 4,50-384$ Wroclaw, Poland 